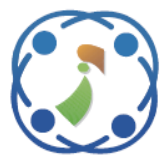

\title{
Optimal Channel Allocation for QoS Provisioning in Cognitive Multihop Radio Adhoc Network
}

\author{
Jaya Lakshmi Arikatla $^{1 *} \quad$ Narasimha Swamy Gavini $^{2} \quad$ Giri Prasad Mahendra Nanjappa $^{1}$ \\ ${ }^{1}$ Department of Electronics and Communication Engineering, \\ Jawaharlal Nehru Technological University Ananthapur, Ananthapuramu, India \\ ${ }^{2}$ Department of Electronics and Instrumentation Engineering, \\ Velagapudi Ramakrishna Siddhartha Engineering College, Andhra Pradesh, India \\ * Corresponding author's Email: Jayalakshmi417@gmail.com
}

\begin{abstract}
Provision quality of service (QoS) is the need of coming generation networks. Wherein mechanism for the provision of quality of service has emerged in different directions, the effort on minimizing the broadcasting overhead in a setup process is observed to be high. The past development in resource allocation has focused on the optimization of quality of service in the communication process. However, not much emphasis is made on the signaling overhead for multicast signal propagation under dynamic network resource condition. The limitation is observed to be more predominant in dynamic network such as mobile Adhoc network. The link overhead under a dynamic resource variation impact on the channel allocation performance and hence introduces delay. This paper, present an approach of resource allocation under the dynamic network condition for 1-hop and Multihop broadcasting. The proposed approach optimizes the channel allocation by the self-detection of available channel due to additional secondary users and allocates a communication channel based on a sensing mechanism. Wherein, the conventional method uses a fixed level of available channel, the proposed approach gives the advantage of channel allocation with channel sensing under dynamic user condition. The proposed approach illustrates a higher success rate and minimization of average delay under a dynamic network condition.
\end{abstract}

Keywords: Mobile adhoc network, Quality of service, Cognitive units, Resource allocation.

\section{Introduction}

In the emergence of new network, cognitive type network has gained a lot attention in recent time. With the advantage of having higher spectrum utilization efficiency, this network are rapidly adapting for the provisioning of high QoS offering in wireless network. The cognitive based network, has given a higher possibility of getting large throughput giving a provision of high QoS provisioning for the next generation upcoming services. To meet the emerging demand of services in mobile devices, different networks are coming together working on an integrated manner to offer long range and high quality of service in resource utilization. In this emergence, a hybrid model of cognitive radio user equipment $(\mathrm{CuE})$ in integration with adhoc network has up come. This network has given a dual benefit of network dynamicity, where the advantage of adhoc network for no infrastructure dependency and adaptive routing provision with dynamic spectrum utilization is used. The integration has the benefit of larger data accessing over a wide range of self-developing network. In the provisioning of Quality of service in such network, various approaches were developed. In [1] a blind estimation approach for network setup using the broadcast operation is developed. The proposed approach defines the broadcasting approach and resource utilization by measuring the success rate and average delay in terms of dynamic allocation of channel slot in the communication. To give a fair allocation of resource, in [2] an inference based resource allocation is suggested. The approach gives the advantage of spectrum efficiency maximization 
with improvising the offered quality of service, when used under a cognitive based network. In [3] a linear programming model in concern to traffic density is presented. The approach defines a new scheduling mechanism in providing the channel. In [4] a weighted mechanism approach is defined for the evolution of spectrum availability and its allocation in a cognitive based wireless network. To observe the effect of network topology in resource allocation, in [5] a topology driven inference based resource allocation approach is defined. The inter cellular interference monitoring is suggested for the coordination of network nodes in resource allocation in different wireless network models. In the provision of quality of service a dynamic scheduling is outlined in [6]. In [7] a spectrum sensing and allocation in cognitive radio is presented. The developed approach allows to use unlicensed user radio spectrum in dynamic approach of user mobility scenario. The developed approach presented a learning based approach in the allocation of spectrum in communication. The packet scheduling scheme is suggested, where aeffort in offering of service compatibility on multimedia service using cell range expansion is suggested. A Bais based model for resource allocation is outlined in [8,9]. A spectrum hole sensing approach and its allocation in cognitive radio is outlined in [10]. The spectrum holes are detected as the underutilized spectrum used in the channel allocation in case of cognitive radio communication. The approach performs a sensing of spectrum hole for the secondary user to optimize the unused spectrum utilization. In a joint effort to have spectral and energy efficiency, in [11] a new spectrum sensing and allocation operation based on the load monitoring and allocated slots for communication is presented. An operational efficiency in terms of offered quality of service is developed. In [12] this efficiency is outlined for a multi-layer radio access network. A radio access technology (RAT) is defined in offering QoS defining multi objective optimization. In [13] an inference governed resource allocation is suggested. The offered QoS is governed interms of link bandwidth, node blockage, packet delivery in a routing node in cognitive based wireless network. In [14] an underlay CRN is considered in developing resource allocation solution. The optimization problem is defined as a global optimization problem and the interference among the primary and secondary users are observed in the utilization of spectrum over the entire network. A modified binary artificial bee colony algorithm is proposed to converge the optimization problem. In [15] a routing approach using a generalized backward utility factor is proposed to offer dynamic resource allocation in QoS frame work for cognitive wireless network. To overcome the spectrum saturation issue in CRN in practical application in [16] a spectrum sensing application is developed over an android smart phone application, where an energy based detector is developed for spectrum sensing taking Bluetooth and Wi-Fi spectrum under consideration. An efficiency of $81 \%$ is observed by the developed approach. An approach defining the multihop scenario in concern to homogenous network and its offering QoS is outlined in [17]. To offer a QoS coding in CRN application, in [18] a priority based scheduling for primary user and secondary user based on the interference model is presented. A resource allocation using priority based coding is developed. The approach defines a dynamic resource allocation (DRAPBS-CRN) approach in cognitive radio application. An efficiency improvement of $26 \%$ is observed. The service oriented QoS provision is outlined in [19], where an internet protocol (IP) based signaling process is defined in provision of end to end packet flow for smoother data flow. In [20], a slot allocation in cognitive based wireless network for the resource allocation is suggested. The overall throughput is concern in provisioning of QoS. An energy model is defined in [21] for the allocation of the spectrum in a cognitive radio network under the noise unsertinity condition. In a multi input multi output (MIMO) communication, spectrum utilization is presented in [22]. The proposed approach apply a co-operative relay in primary traffic to develop channel allocation. A priority based scheduling approach is defined to optimize resource allocation in constraint to fairness, minimum rate guarantee and minimum delay guarantee. The solution outlined is developed with minimum primary user interference. In $[23,24]$ a queue based mechanism is proposed, where the resource allocation is developed for a heterogeneous network under the constraint of delay consideration. The approach defines a channel allocation based ion the demanded service and the distance of each of the user giving different queue order to different service demands. The selfish greedy approach of spectrum utilization is outlined in [25]. The issue of spectrum blockage and channel consumption in a greedy manner is focused. In the optimization of spectrum utilization this issue is observed as a limiting constraint due to blockage of unused channel utilization by fake signal attack. To overcome a collaborative mode of channel allocation approach is presented. In past developments made in resource allocation majority of the work focused on the 
optimization of spectrum sensing based on the available primary and secondary users and their spectrum availability methods were developed in sensing the spectrum and allocation of spectrum based on interference model and energy detection model. This CRN has given the advantage of utilizing the unutilized spectrum $\mathrm{n}$ a more efficient manner than other communication system. However, the CRN is analyzed with an objective of spectrum optimization by improvising the spectrum sensing and its allocation accuracy, not much work is observed in terms of network and the dynamicity in user mobility. In next generation communication network, multiple networks are integrated to form a heterogeneous network to offer wide range of service oriented communication. With this perspective in this work CRN and Ad-Hoc network are integrated to evaluate the communication performance. Wherein CRN offers an optimal spectrum sensing, Adhoc network offers a dynamic spectrum availability. Users in Adhoc network moves in a random manner which introduces a dynamic spectrum condition in the network. It is required to develop a new approach in handing the dynamic spectrum variation in such network. An addition or removal of a node offers an extra spectrum or deletion which should be sensed and allocated or removed accordingly. A in proper sensing leads to high delay in channel allocation or wastage of available spectrum, minimizing the network efficiency. Hence, in this paper, a new approach of spectrum sensing and its allocation based on the dynamic movement of the user is evaluated. A new channel allocation approach based on the sensing approach is developed, which minimizes the error probability by optimizing the MSR problem with a new derived cost function.

To present the suggested approach, the rest of the paper is outlined in 6 sections. Wherein section 2 outline the system model for a cognitive based Adhoc network, section 3 outlines the conventional QB2IC approach in provisioning quality of service in this network. The proposed approach of spectrum allocation in concern to interference and node dynamicity is outlined in section 4. Section 5 illustrates the simulation results obtained for the developed approach, wherein section 6 presents the conclusion of this paper.

\section{System model}

In the evaluation of a network model, a randomly distributed wireless adhoc network is used. The system outline of the network is illustrated in Fig. 1. The network scenario illustrates a randomly distributed network where the nodes are placed at a random coordinates in the network. The network is defined with an arbitrary primary (PU) and secondary user (SU). Wherein the network is defied in random format, the nodes are constraint with their communication range as illustrated with the circle marked.

This network is defied with all nodes having cognitive property called cognitive user equipment $(\mathrm{CuE})$, and the nodes are AdHoc enabled to selfcreate a network with the process of neighbor discovery and route establishment. In the process of communication, each of the node $\left(N_{i}\right)$, where $i=1 \ldots n$, and ' $n$ ' defines number of nodes are used to broadcast a link request packet to their neighbor. The nodes falling within the communication range $\left(R_{i}\right)$ as marked with a circle region acknowledge the request to build a 1 hop link. The process is repeated for all the nods in the network, and a set of 1 hop links are registered at each node. In the analysis of broadcasting effect, in this work two summation scenario of 1 hop and multi-hop network is considered. The process of 1-hop and multi hop is illustrated in Fig. 2.

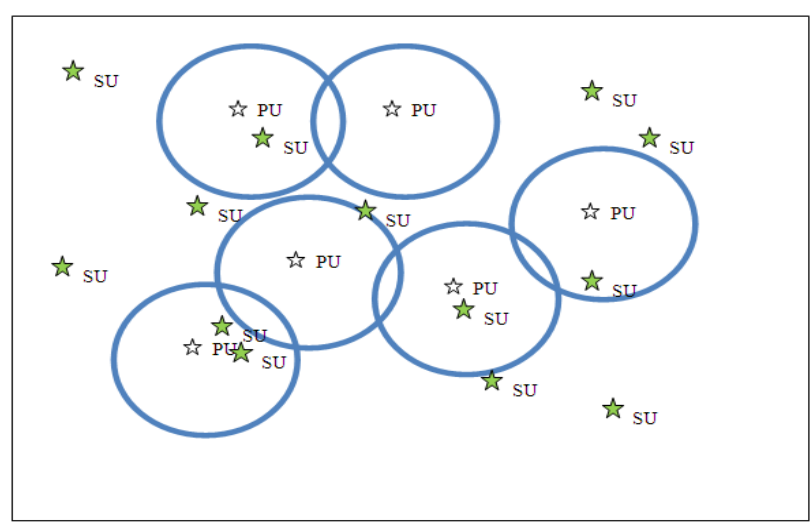

Figure. 1 Network scenario

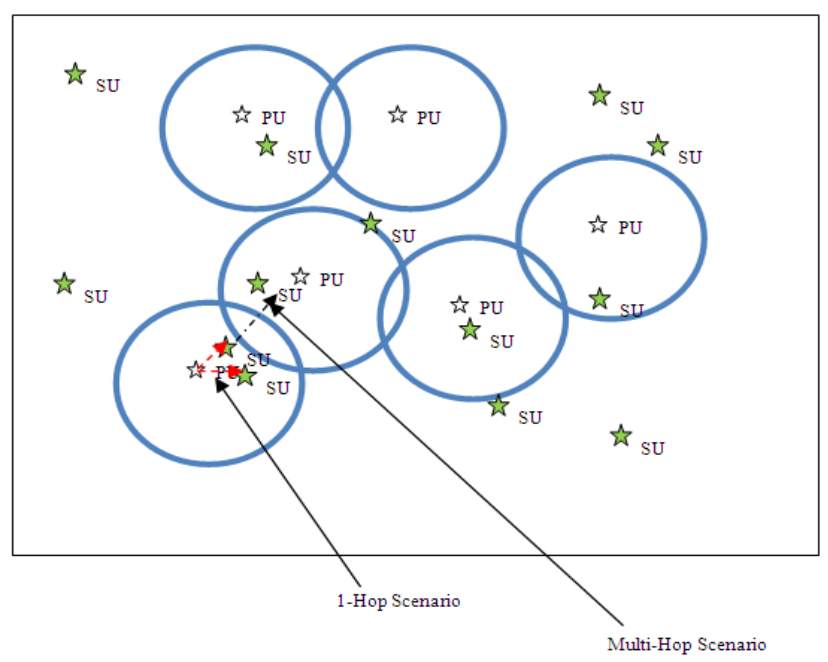

Figure. 2 Communication scenario 
In the broadcasting scenario, the nodes within a direct link are test for the resource allocation in a random selection mode and a full sequential mode as outlined in next section. The nodes falling out of the communication range and are linked via an intermediate link fall under a multi-hop case, where the node is requested or acknowledge via intermediate link nodes. To provision of quality of service in this network, a blind quality of service provision in broadcast is outlined in [1]. The suggested approach for quality provisioning is outlined in next section.

\section{QB ${ }^{2} \mathrm{IC}$ : QoS broadcast protocol [1]}

In the provision of quality of service in a mobile adhoc network with cognitive equipment in [1] QoS broadcast protocol $\left(\mathrm{QB}^{2} \mathrm{IC}\right)$ under blind information is suggested. The approach defines a blind information exchange is building a link where the request is blindly broadcast over the network. In the cognitive network where the spectrum is heterogeneous in nature, the signalling overhead is observed to be dynamic; the allocated channel in this scenario must be properly controlled to have efficient data change. In the suggested approach, the resource allocation under a single hop and a multihop network is proposed. The network, validate the resource allocation interim of allocated spectrum and selective slot mechanism. For a given network the allocated slot $S^{t}$ at any instance of time $t$ is given by,

$$
S^{t}=\frac{\sum_{n \in N} S_{n}^{t}}{N}
$$

Where $n$ is the number of active user within a communication range, and $N$ are the total number of nodes in a network. The active number of slots allocated is defined by,

$$
S_{n}^{t}=\sum_{r \in R} U_{n}^{t} \times S_{n}^{t}
$$

where $R$ is the set of PU/SU registered, and $a \in A$ is the radio offered to $A$ at time $t$. where $R$ is define as a registered set of nodes, and $\mathrm{r}$ is the observing node within this set. $U_{n}^{t}$ is the $\mathrm{CuE}$ for which a $S_{n}^{t}$ slots are allotted. At any instance of time, the total slot allotted at a node is observed as the aggregated slots from the entire registered user within the communication range.

In the broadcasting process, the channels are selected based on either a random mode selection of full selection model. In the random mode selection

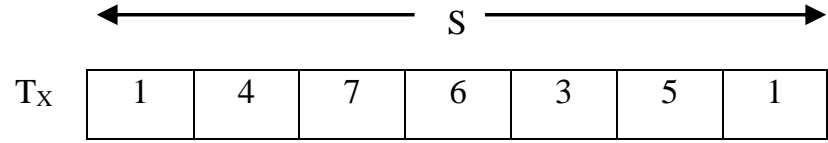

Figure. 3 Illustration of channels in a random allocation

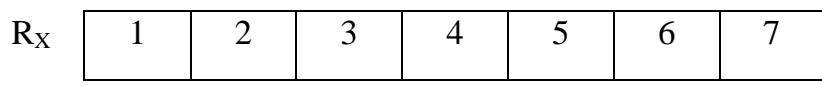

Figure. 4 Channel selection scheme in full selection mode

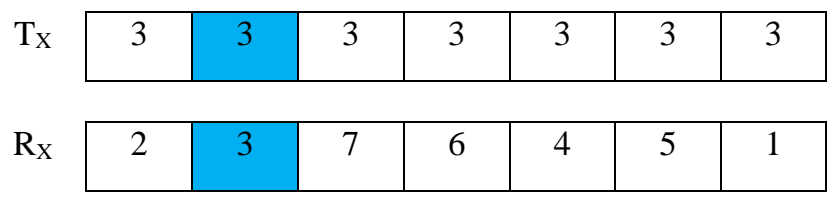

Figure. 5 Test synchronization pattern

the channels are randomly picked whereas full selective mode, the channels are sequentially been picked. The approach of channel selection is illustrated in figure below.

Here the channels are selected based on the availability is defined for a period observation of 1 communication beacon. Wherein the observation under multi-hop scenario as the network is dynamic, the nodes are adaptively been updated in each beacon period. The node inclusion or exclusion are not been considered in the conventional modeling. The searching overhead of the synchronizing channel is measured as the average delay and the selection of a search for a synchronizing channel is termed as the successes rate. This parameter defines the overhead in channel synchronization under different registered channel slot.

For a given channel slot, as illustrated in Fig. 5, the synchronization effort to synchronize a channel 3 would be 5 in case of random slot selection based on the pattern illustrated in Fig. 3, whereas the search effort will be 3 in case of a full selection approach.

Wherein the search effort defines the success rate and the probability of slot synchronization defines the average delay of the network, the resource dynamicity, i.e., the available number of slots in the network can also impact on the performance. As the network is dynamic due to Adhoc nature, and the nodes are cognitive type, the spectrum availability is dynamic, and hence the slots are dynamically varied. In consideration of this factor, the success rate is probable to be improved.

\section{Proposed adaptive control method}

In the proposing approach, the adaptation of add-on users due to adhoc nodes is analyzed. An illustration of the user's updation in the network is illustrated in figure 6. The add-on user SU+ are 


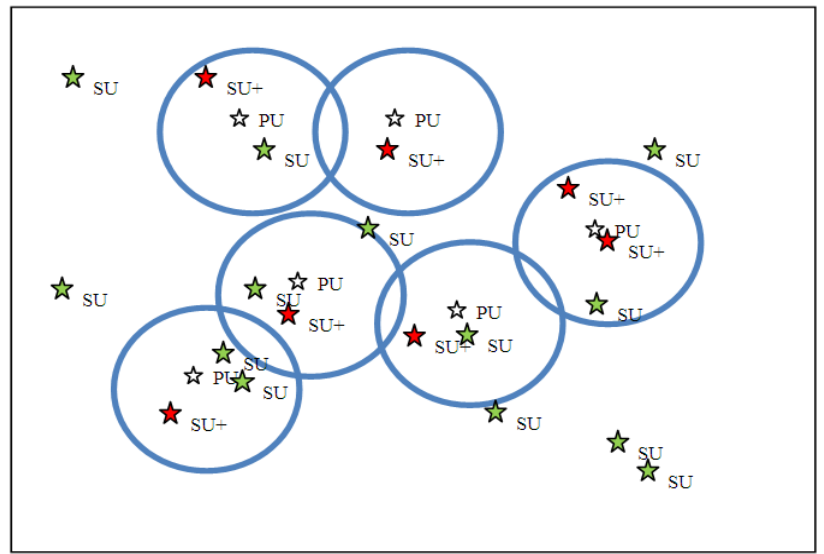

Figure. 6 SU updating scenario

randomly get added to the network, which are observed to give additional spectrum availability to the existing network.

In the updation of secondary spectrum, the allocation of the spectrum is based on the demanded service and the available resources. The PU/SU allocation depends on the demanded service and the node performs a spectrum sensing operation to drive the available resource. In the process of spectrum allocation, the allocation is dynamically been engaged or released based on the availability and demanded service. In the process of spectrum sensing or updation, the slots area dynamically been switched and the sensed slot synchronized between the transmitting and receiver node is selected in either a random selection mode or a full sequential mode. For an initial slot allocation of $s \in S$, where $\mathrm{s}$ is the allocated slot and $S$ is the total slots, wherein the slot allocation $S^{t}$ is observed to updated with the allocation or release of a channel. The updation of the spectrum slots is then defined as,

$$
S_{a v 1}^{t}=\sum_{n \in N} S_{n}^{t}-s_{i}^{t}
$$

Where $\sum_{n \in N} S_{n}^{t}$ is the aggregated sum of all the spectrum slots sensed. Each $\mathrm{CuE}$ device process a spectrum sensing operation, based on the approach of energy detection model [21], defined by;

$$
E_{t h}=\sum_{i=1}^{n}\left(\frac{r_{i}}{\sigma}\right)^{P}
$$

Where $r_{i}$ is the recived signal wuth a noise variance of $\sigma$. The energy threshold for the spectrum sensing is given by eqn. 4 , where ' $P$ ' is an amplifying factor governing to the probability of channel variations, given as $\mathrm{P}=\{3,10\}$. In the process of spectrum swing, the estimated energy of the received signal is compared with the derived energy thresholds, and a signal having energy higher than this threshold is taken as a occupied channel, else the system consider it as a free channel.

$$
\text { Avl_Spec }=\left\{\begin{array}{rr}
1 ; & E_{\text {th }} \geq E_{\text {measure }} \\
0 ; & \text { Otherwise }
\end{array}\right.
$$

The total available spectrum is then given by,

$$
S_{a v 1+}^{t}=\sum_{n \in N} S_{n}^{t}+s_{a v l+}^{t}
$$

Where, $s_{a v l+}^{t}$ is the sensed spectrum for the Avl_Spec=1.

In the allocation of this spectrum, the success rate will be improved as the available spectrum is increased. However, it is observed that, a random allocation of this available SU+ spectrum could lead to higher interference as the channel is added with a higher volume of data. It is hence, optimized as a trade-off for the service offered over the success rate. This problem is solved as a maximum spectrum rate (MSR) allocation problem. This approach optimizes the spectrum allocation based on the constraint of minimal interference and maximum resource allocation. A cost function is then defined given as,

$$
s_{\text {alloc }}^{t}=\min _{\{n\}}\{I\} \Rightarrow s_{\text {avl+ }}^{t} \leq S_{a v 1+}^{t}
$$

Here, from the sensed spectrum, the allocable spectrum $s_{\text {alloc }}^{t}$ is constraint with the interference minimization problem. It is computed for the available spectrum to observe the interference occurrence, and for the $s_{\text {alloc }}^{t}$ giving minimum interference $\min _{\{n\}}\{I\}$ is selected. This allocation guarantees the offering quality of service, with higher spectrum allocation. The proposed spectrum updation approach is outlined in the given algorithm below,

Algorithm:
Sense the available spectrum $s_{\text {avl+ }}^{\mathrm{t}}$ for the period t;
Forward a packet L;
Calculate the Interference Energy $\left(E_{\text {int }}\right)$
$\quad$ if $\left(E_{\text {int }}<E_{\text {th }}\right)$
Generate a channel selection number $\mu$;
if $\left(s_{i}^{t}=\mu\right)$
$\quad$ synchronize the channel;
else
End


Wherein in the scenario of random channel allocation and additional spectrum result in additional channel which can be allocated for data exchange resulting in higher success rate. However, the pooling of channel allocation in a random allocation is higher resulting in delay. This is observed to be lower in full selection approach.

\section{Simulation result}

To validate the proposed approach, a simulation model is developed in Matlab environment. A randomly distribute network is created with each node having a cognitive property. To evaluates the performance of the proposed approach, the developed approach is compared under 4 simulation scenario here the network is tested for single hop, multihop network, with random and full sequence search scheme. The simulation parameters are compared for the $\mathrm{QB}^{2} \mathrm{IC}$ model [1] and the proposed inference governed adaptive control method (IAC) to evaluate the performances. The success rate and the average delay for the network is computed. Where, the success rate $(S R)$ is defined as,

$$
S R=\frac{\text { No.ofpacketSuccesfullyreceived }}{\text { Total No.ofpacketgenerated }}
$$

The simulation parameter for the network considered is given in Table 1 below.

The simulation is performed under two cases, wherein constant network models is considered to evaluate the two methods and in second case the node density are varied to observe the performance of variation on the spectrum efficiency. The observed simulation observations are as illustrated in Fig. 7.

The nodes are randomly placed with the $x, y-$ coordinates randomly scattered for number of nodes. The network area is taken as $30 \times 30$ for the node placement. The success rate and the average delay observed for the developed network is illustrated in Figs. 8 and 9 , respectively.

Table1. Network parameter in simulation

\begin{tabular}{cc} 
Network Details & Parameters \\
\hline Node distribution & Random \\
No. of PU & 10 \\
No. of SU & 20 \\
Network area & $30 \times 30$ \\
Number of channel & 10 \\
Communication range & 10 \\
Interference margin & 0.15 \\
Network variability rate & 5 \\
\hline
\end{tabular}

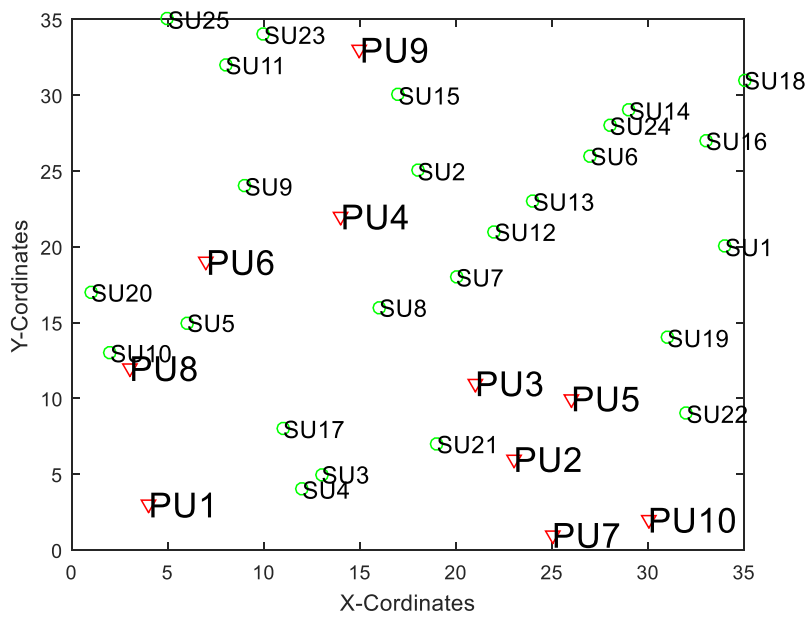

Figure. 7 Deployed PU/SU nodes in a random distribution with 30 nodes

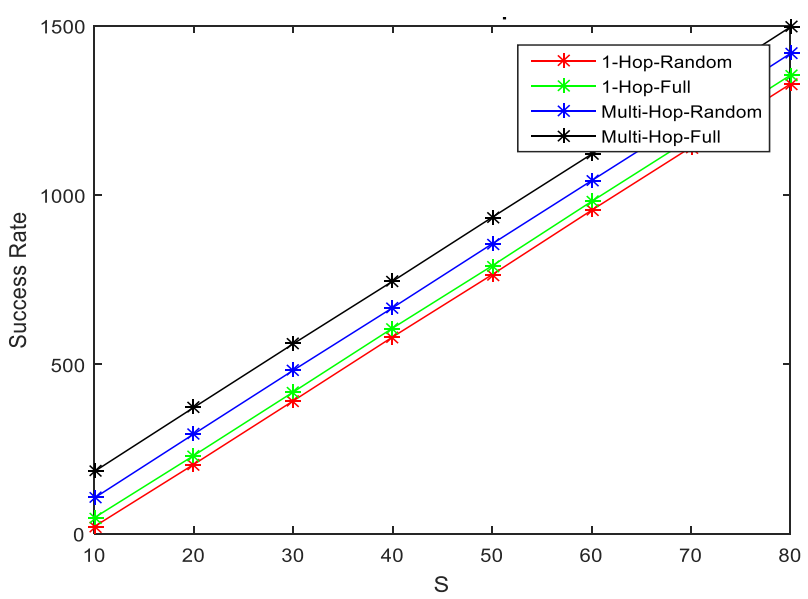

Figure. 8 Success rate over different slot availably using $\mathrm{QB}^{2} \mathrm{IC}$ approach

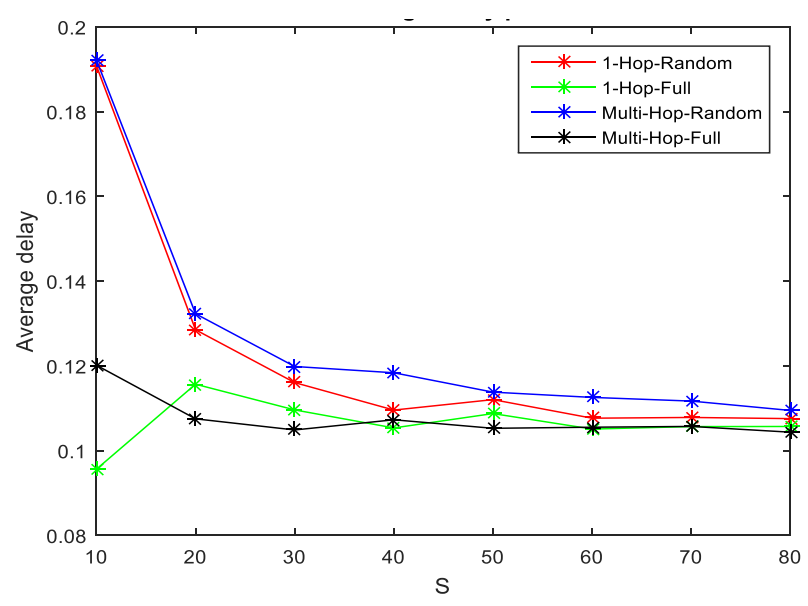

Figure. 9 Average delay over different slot availably using QB ${ }^{2} \mathrm{IC}$ approach

The success rate for a full search multi hop scenario is observed to be higher, due to a faster slot synchronization and higher degree of slot availability. The average delay for this method is also observed to be lower. The observation for the 
proposed method for a 30 node network is presented in Figs. 10 and 11, respectively.

The success rate for the proposed method is observed to be enhanced by 1500 as compared to the $\mathrm{QB}^{2} \mathrm{IC}$ method. An optimal spectrum sensing result in optimal channel allocation giving a higher success rate. The average delay in this case is reduced by a factor $0.1 \mathrm{sec}$. To validate the performance of proposed approach for the node dynamicity in the network 10 nodes are randomly added to the existing network. These added nodes gives an opportunity of additional spectrum in the network. The objective is to optimally sense this spectrum and utilize under resource allocation approach. The two developed methods are simulated over the network and the observations are made to measure the efficiency of the network. The obtained observations are as illustrated below.

The developed system is observed to improve the success rate performance based on the allocated slots for channel allocation. The average delay and the success rate is measured as a quality metric in defining the operational efficiency of the developed system. The success rate of the proposed approach is

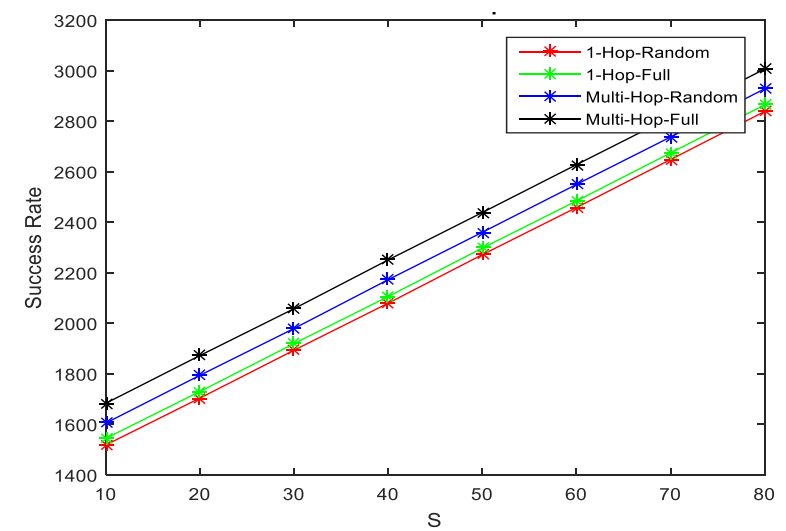

Figure. 10 Success rate over different slot availably using IAC approach

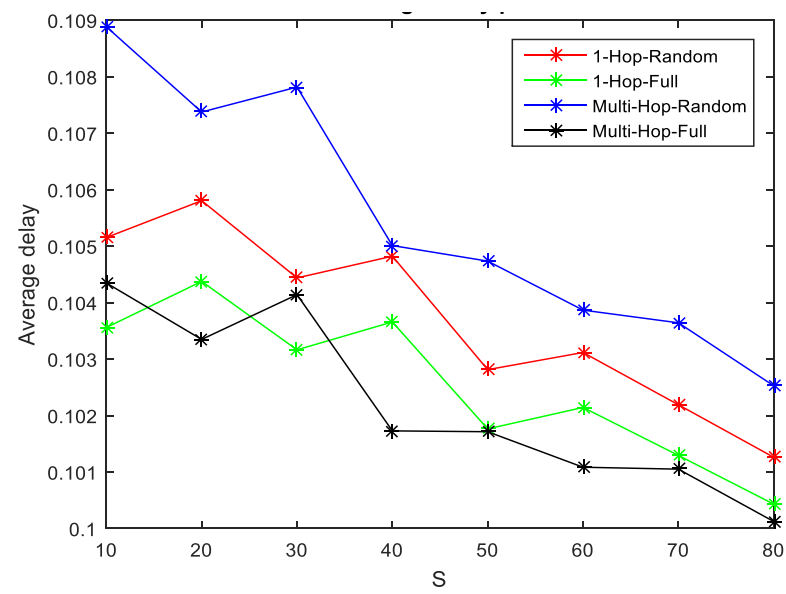

Figure. 11 Average delay over different slot availably using proposed IAC approach observed to be 1500 packets higher than the conventional QB ${ }^{2} \mathrm{IC}$ approach for a 30-node network in a 1-hop random searching scheme. Wherein, it is 1510 packets in a Multi-hop random search scheme. The proposed approach has a 1520 packets increase in success rate in a 40 node density. The observations of the developed approach in a 1hop and multi hop search scheme illustrate an increment in the success rate.

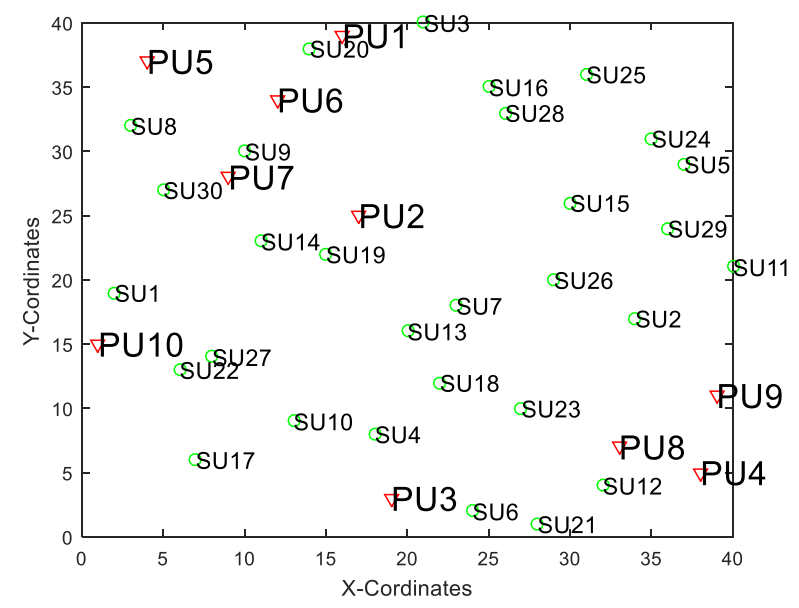

Figure. 12 Deployed PU/SU nodes in a random distribution with 40 nodes

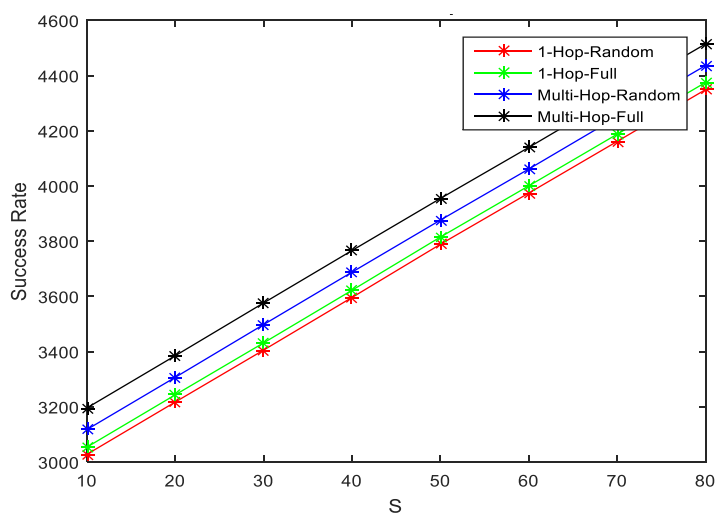

Figure. 13 Success rate over different slot availably using $\mathrm{QB}^{2} \mathrm{IC}$ approach

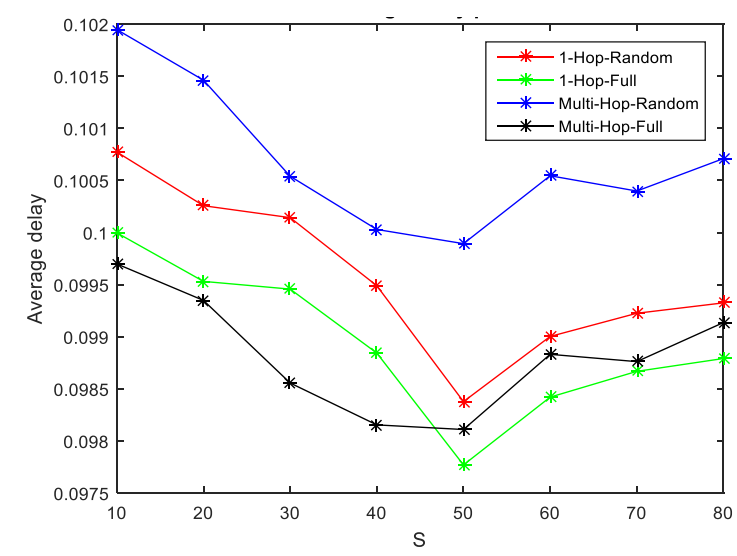

Figure. 14 Average delay over different slot availably using QB ${ }^{2} \mathrm{IC}$ approach 
Table 1. Comparative analysis of the developed approaches for success rate

\begin{tabular}{|c|c|c|c|c|c|}
\hline Node Density & Method & \multicolumn{2}{|c|}{ 1-hop } & \multicolumn{2}{c|}{ Multi-hop } \\
\hline \multirow{3}{*}{30} & \multirow{2}{*}{ QB $^{2}$ IC [1] } & Random & Full & Random & Full \\
\cline { 2 - 6 } & & 1300 & 1360 & 1400 & 1500 \\
\cline { 2 - 6 } & Proposed Approach & 2810 & 2860 & 2910 & 3000 \\
\hline \multirow{2}{*}{40} & QB $^{2}$ IC [1] & 4370 & 4428 & 4550 & 4650 \\
\cline { 2 - 6 } & Proposed Approach & 5850 & 5916 & 6030 & 6119 \\
\hline
\end{tabular}

Table 2. Comparative analysis of the developed approaches for average delay in sec.

\begin{tabular}{|c|c|c|c|c|c|}
\hline Node Density & Method & \multicolumn{2}{|c|}{ 1-hop } & \multicolumn{2}{c|}{ Multi-hop } \\
\hline \multirow{3}{*}{30} & \multirow{2}{*}{ QB $^{2}$ IC [1] } & Random & Full & Random & Full \\
\cline { 2 - 6 } & & 0.153 & 0.15 & 0.151 & 0.13 \\
\cline { 2 - 6 } & Proposed Approach & 0.103 & 0.103 & 0.13 & 0.09 \\
\hline \multirow{2}{*}{40} & QB $^{2}$ IC [1] & 0.09 & 0.085 & 0.088 & 0.081 \\
\cline { 2 - 6 } & Proposed Approach & 0.065 & 0.05 & 0.049 & 0.044 \\
\hline
\end{tabular}

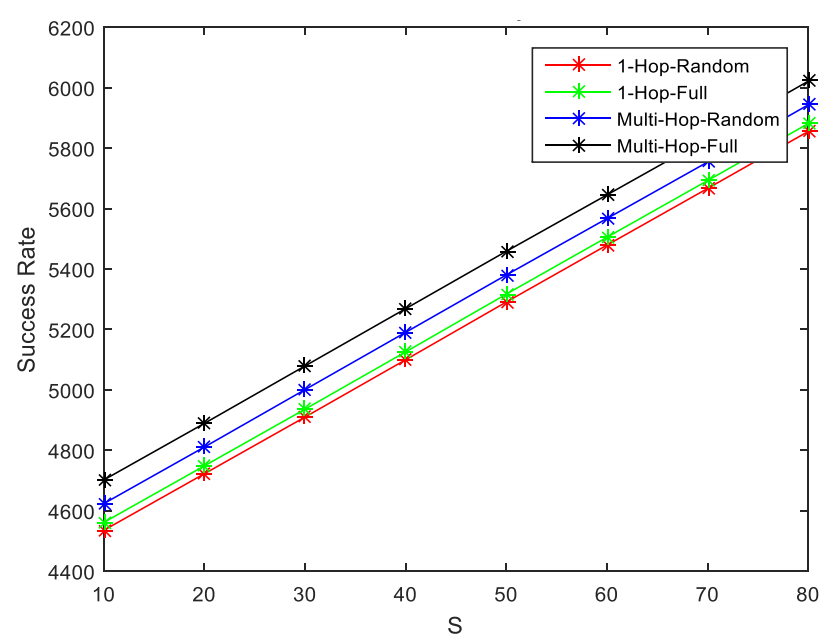

Figure. 15 Success rate over different slot availably using IAC approach

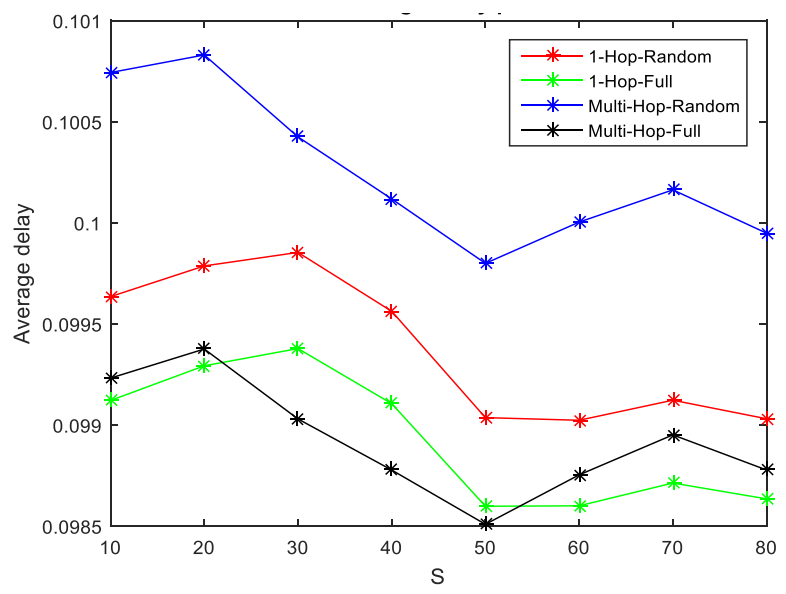

Figure.16 Average delay over different slot availably using IAC approach

The improvement is observed due to an additional spectrum sensing and allocation in the proposed approach. The comparative delay is observed to be reduced by $0.12 \mathrm{Sec}$. The lower blockage of data results in a faster clearance, minimizing the communication overhead and hence intern reduces the delay offered.

\section{Conclusion}

This paper presented a new approach of spectrum updation scheme in a mobile Adhoc network with cognitive devices. The approach defines a new approach of spectrum allocation based on the availability of spectrum sensed and the inference margin. The suggested approach minimizes the error probability by optimizing the MSR problem with a new derived cost function. The suggested approach gives and enhancement in success rate and minimization in delay factor as observed form the simulation result. The optimal spectrum allocation improves the slot probability and enhances the network performance. The simulation results obtained from the developed approaches illustrates the improvement in the obtained success rate. An additional user in the network range leads to higher spectrum availability. A proper spectrum sensing leads to increase in the available data rate, which intern results in more available channels. The additional channel gives a higher search performance and minimizes the delay constraint. The approach illustrates a higher success rate of packet delivery, which makes the proposed approach more compatible to various real time usages. The channel allocation effort minimized in the proposed approach, has the possibility to improve by the consideration of data accessing and user mobility under communication scenario after the channel allocation. The data overhead, and link interference could leads to blockage in communication phase, which could be minimized via an optimal resource allocation during data exchange. 


\section{References}

[1] Y. Song and J. Xie, "QB" IC: A QoS-Based Broadcast Protocol Under Blind Information for Multihop Cognitive Radio Ad Hoc Networks", IEEE Transactions On Vehicular Technology, Vol. 63, No. 3, pp 1980-1997, 2014.

[2] S. S. Krishna, S. Chatzinotas, and B. Ottersten, "Interference alignment for spectral coexistence of heterogeneous networks", EURASIP Journal on Wireless Communications and Networking, Vol.21, No.6, pp 1-14, 2013.

[3] S. V. Hanly, C. Liu, and P. Whiting, "Capacity and stable scheduling in heterogeneous wireless networks", IEEE Journal on Selected Areas in Communications, Vol 33, No.6, pp 1266-1279, 2015.

[4] T. H. Mohamad, A. H. Al-Ghushami, and Z. R. Yahya, "Selection of access network using cost function method in heterogeneous wireless network", In: Proc. of International Conf. on Multimedia Computing and Systems, pp 777782, 2014.

[5] A. Khandekar, N. Bhushan, J. Tingfang, and V. Vanghi, "LTE-advanced: Heterogeneous networks", In Proc. of International Conf. on European Wireless Conference, pp 978-982, 2010.

[6] W. Mansouri, K. Mnif, F. Zarai, and M. S. Obaidat, "A new multi-rat scheduling algorithm for Heterogeneous wireless networks", Journal of Systems and Software, Vol 31, No.7, pp 1857-1875, 2015.

[7] P. Rungsawang and A. Khawne, "The implementation of spectrum sensing and spectrum allocation on cognitive radio", In: Proc. of International Conf. on Advanced Communication Technology, 2017.

[8] L. Mengting, Y. Teng, and M. Song, "Tradeoff Analysis between Spectrum Efficiency and Energy Efficiency in Heterogeneous Networks (HetNets) Using Bias Factor", Journal of Communications, Vol. 10, No.8, pp 2557-2570, 2015.

[9] S. Kyuho, E. Oh, and B. K. Chari, "Energyefficient design of heterogeneous cellular networks from deployment to operation", Journal of Computer Networks, Vol.78, No. 6, pp 95-106, 2015.

[10] E. A. Adaba, E. H. Abdelhy, and F. W. Zaki, "Cognitive Radio Spectrum Sensing and Secondary User Allocation", International Journal of Innovative Research in Computer and Communication Engineering, Vol. 5, No. 1, 2017.

[11] H. R. Qingyang and Y. Qian, "An energy efficient and spectrum efficient wireless heterogeneous network framework for $5 \mathrm{G}$ systems", IEEE

Communications Magazine, Vol. 52, No. 5, pp 94-101, 2014.

[12] J. B. Rao, and A. O. Fapojuwo, "Analysis of Spectrum Efficiency and Energy Efficiency of Heterogeneous Wireless Networks With Intra/Inter-RAT Offloading", IEEE Transactions on Vehicular Technology, Vol.64, No.7, pp 31203139, 2015.

[13] S. Hua, L. Jiandong, Z. Li, and J. Honghao, "Dynamic spectrum allocation in heterogeneous wireless networks under interference constraints across the cell coverage", Wireless Communications and Mobile Computing, Vol. 15, No. 7, pp 11881197, 2015.

[14] X. Zhang, X. Zhang, L. Han, and R. Xing, "Utilization-Oriented Spectrum Allocation in an Underlay Cognitive Radio Network", IEEE Access, Vol.6, pp. 12905-12912, 2018.

[15] L. Badia, M. Miozzo, M. Rossi, and M. Zorzi, "Routing schemes in heterogeneous wireless networks based on access advertisement and backward utilities for QoS support [Quality of Service based Routing Algorithms for Heterogeneous Networks]", IEEE Communications Magazine, Vol. 45, No. 2, pp 67-73, 2007.

[16] V. Vijayasarveswari, S. Khatun, M. M. Fakir, M. N. Nayeem, L. M. Kamarudin, and A. Jakaria, "Cognitive radio based optimal channel sensing and resources allocation" In: Proc. of International Conf. AIP, Vol.1808, No.2, 2017.

[17] F. Richter, A. Fehske, P. Marsch, and G. Fettweis, "Traffic demand and energy efficiency in heterogeneous cellular mobile radio networks", In: Proc. of International Conf. on Vehicular Technology, pp. 1-6, 2010.

[18] T. Santhamurthy and K. Parasuramane, "Appropriate Channel Selection for Dynamic Resource Allocation with Priority Scheduling Approach in Multi-hop Cognitive Radio Networks", International Journal of Intelligent Engineering and Systems, Vol. 10, No. 2, pp. 110, 2017.

[19] G. Aristomenopoulos, T. Kastrinogiannis, Z. Li, and S. Papavassiliou, "An autonomic Qoscentric architecture for integrated heterogeneous wireless networks', Journal of Mobile Networks and Applications, Vol.16, No. 4, pp 490-504, 2013. 
[20] D. Zeyang, J. Liu, and C. Wang, "QoS-based device-to-device communication schemes in heterogeneous wireless networks", IET Communications, Vol. 9, No. 3, pp. 335-341, 2015.

[21] C. J. Christopher, B. B. Reddy, and D. S. Emmanuel, "An Energy-Efficient Cooperative Spectrum Sensing Strategy with Robustness Against Noise Uncertainty for Cognitive Radio Networks", Arabian Journal for Science and Engineering, Vol. 41, No. 9, pp. 3399- 3405, 2016.

[22] T. Santhamurthy and K. Parasuraman, "EPBDRA: Efficient Priority Based Dynamic Resource Allocation in Heterogeneous MIMO Cognitive Radio Networks", International Journal of Intelligent Engineering and Systems, Vol. 11, No. 1, pp. 276-285, 2018.

[23] B. S. Awoyemi, B. T. Maharaj, and A. S. Alfa, "Resource Allocation in Heterogeneous Buffered Cognitive Radio Networks", Wireless Communications and Mobile Computing, Vol. 2017, 2017.

[24] D. Tamilarasan, K. Balakrishnan, and H. M. Manjula, "Efficiency Enhancement in Channel Allocation in Cognitive Radio Network using Priority Scheduling", International Journal of Innovations \& Advancement in Computer Science, Vol.6, No.3, pp. 70-75, 2017.

[25] K. Shinde, S. Jyoti, and H. Patil, "Performance Evolution of CR-AD HOC Network by Updated Joint Cooperative Spectrum Sensing Technique", International Journal of Scientific Research in Science and Technology, Vol.3, No.3, 2017. 